\title{
Clinical Utilization and Cost of Thrombophilia Testing in Patients with Venous Thromboembolism
}

\author{
Manila Gaddh ${ }^{1}$ En Cheng ${ }^{2}$ Maha A.T. Elsebaie ${ }^{1}$ Imre Bodó ${ }^{1,3}$ \\ ${ }^{1}$ Department of Hematology and Medical Oncology, Emory \\ University School of Medicine, Atlanta, Georgia, United States \\ 2 Department of Chronic Disease Epidemiology, Yale University \\ School of Public Health, New Haven, Connecticut, United States \\ ${ }^{3}$ Department of Medicine and Hematology, Semmelweis University, \\ Budapest, Hungary
}

TH Open 2020;4:e153-e162.

\author{
Address for correspondence Manila Gaddh, MD, 1365 C. Clifton Road \\ NE, Atlanta, GA 30322, United States \\ (e-mail: manila.gaddh@emory.edu).
}

\begin{abstract}
Keywords

- venous thromboembolism

- venous thrombosis

- costs and cost analysis

- thrombophilia

- hypercoagulability

Introduction Testing for inherited and acquired thrombophilias adds to the cost of care of patients with venous thromboembolism (VTE), though results may not influence patient management.

Methods This is a single-center, retrospective study conducted at Emory University Hospitals from January to December 2015 to (1) determine the pattern of thrombophilia testing in patients with VTE, (2) study the impact of results of thrombophilia testing on clinical decision-making, and (3) determine the direct costs of thrombophilia testing in patients with VTE.

Results Of the 266 eligible patients, 189 (71\%) underwent testing; 51 (26.9\%) tested positive and the results impacted management in $32(16.9 \%)$ of tested patients. Patient undergoing testing were more likely to be younger than 40 years ( 30.9 vs. $18.2 \%$ ), have had prior pregnancy loss ( 9.0 vs. $0 \%$ ), or known family history of hypercoagulability ( 24.9 vs. $10.4 \%$ ), and were less likely to have had provoked VTE ( 37 vs. $79.2 \%)$. The most common thrombophilias tested were antiphospholipid syndrome (60.1\%), factor $\mathrm{V}$ Leiden (59.7\%), and prothrombin gene mutation (57.5\%). Direct costs of thrombophilia testing were $\$ 2,364.32$ per patient, $\$ 12,331.55$ to diagnose 1 positive, and $\$ 19,653.41$ per patient-management affected.

Conclusion We noted significant variability in selection of patients and panel of tests, sparse utilization of test results in patient management, but high cost associated with thrombophilia testing in patients with VTE. With guidelines advocating selective use of thrombophilia testing and attention to potential impact of test results in patient management, we propose the need for measures at institutional levels to improve testordering practices.
\end{abstract}

\section{Introduction}

Since the discovery of antithrombin (AT) deficiency as an inherited thrombophilia in 1965 , several inherited and acquired thrombophilias have been described as risk factors for venous thromboembolism (VTE). ${ }^{1}$ As far as VTE management is con- cerned, the role of thrombophilias in determining the duration or choice of anticoagulant remains uncertain. ${ }^{2,3}$ In everyday practice, however, physicians and patients are often inclined to request thrombophilia testing in the hope of (1) finding a predisposing cause for VTE, (2) understanding the patients' received

February 7, 2020 accepted after revision June 15,2020
DOI https://doi.org/

10.1055/s-0040-1714334. ISSN 2512-9465.
๑) 2020 Georg Thieme Verlag KG
Stuttgart · New York

License terms

(c) (i) 
risk of VTE recurrence, (3) estimating VTE risk for family members, and (4) obtaining information that would help optimize management.

There is no defined panel of thrombophilia testing endorsed by guidelines. ${ }^{4}$ Moreover, physicians are directed to determine duration of anticoagulation for an individual patient based on an assessment of the patient's risk for recurrent VTE and bleeding. ${ }^{5}$ British and National Institute for Health and Care Excellence guidelines go on to suggest using thrombophilia testing only if it is determined that the results will impact patient management. ${ }^{6}$ There is considerable heterogeneity in the relative risk of recurrence associated with individual thrombophilias reported in literature. ${ }^{7,8}$ Determining the role of thrombophilia itself in the occurrence or recurrence of VTE in an individual patient is further complicated by the fact that multiple intrinsic and situational factors such as age, gender, body mass index, pregnancy, and postoperative state may interact variably with the underlying thrombophilia to manifest a thrombotic event. Limited data exist on the comparative effectiveness of different classes of anticoagulants in patients with underlying thrombophilia. A recent systematic review and meta-analysis suggests superiority of vitamin $\mathrm{K}$ antagonists over direct oral anticoagulants (DOACs) in patients with high-risk antiphospholipid syndrome (APS), while reporting equivalent efficacy and safety of these treatment options in the rest of the thrombophilias. ${ }^{9,10}$

In these circumstances, lack of specific guidance from academic societies regarding adaptation of thrombophilia testing in clinical practice can lead to significant variability in what tests are ordered, when they are ordered, and how they are interpreted. While the results may not add value to patient management, the tests certainly increase the cost of management of venous thromboembolic disorders. ${ }^{11}$

We performed this study to explore the pattern of thrombophilia testing, impact of the thrombophilia workup results on clinical management decisions, and direct cost of such tests in patients with VTE at our tertiary care center.

\section{Materials and Methods}

\section{Study Design}

This is a single-center, retrospective study conducted at Emory University Hospitals with the following objectives: (1) determine the pattern of thrombophilia testing in patients with VTE, (2) study the impact of results of thrombophilia testing on clinical decision-making, and (3) determine the direct costs of thrombophilia testing in patients with VTE. The study was approved and a waiver of patient informed consent was granted by Emory University Institutional Review Board (IRB).

\section{Patient Identification}

The Hematology Service at Emory University Hospitals maintains an IRB-approved database for all patients seen by the Hematology Service in the inpatient or outpatient settings. From the Emory Hematology Service database, we identified adult patients, who were seen by Emory Hematology for the evaluation and treatment of VTE between January and December 2015 in the inpatient or outpatient settings. Exclusion criteria included: (1) no formal evaluation by the Emory Hematology Service (e.g., patients never showed up to any of their appointments), (2) insufficient information on VTE event in patient chart, (3) no history of VTE, or (4) superficial venous thrombosis only.

\section{Data Extraction}

For eligible patients, electronic medical records (EMRs) were reviewed for data related to sociodemographics, medical history, details of thromboembolic events, thrombophilia workup, and patient management ( - Table 1). Patient data was extracted manually into predesigned case report forms, then deidentified and anonymized prior to analysis.

We categorized the most recent VTE event at the time of initial hematology consult as our index episode. The following VTE episodes were classified as "provoked" per the International Society of Thrombosis and Haemostasis definition: (1) VTE occurring within 3 months of surgery with general anesthesia, cesarean section, prolonged hospital stay, estrogen therapy, or prolonged immobility, and (2) VTE associated with an indwelling venous catheter or underlying cancer. ${ }^{12}$

\section{Thrombophilia Testing}

All tests ordered by treating physicians as part of thrombophilia workup were included for description of the pattern of thrombophilia testing. Thrombophilia workup was defined

Table 1 Data collected in case report forms

\begin{tabular}{|l|l|}
\hline $\begin{array}{l}\text { Demographic } \\
\text { data }\end{array}$ & $\begin{array}{l}\text { Age at time of evaluation } \\
\text { Gender, race } \\
\text { Height, weight, and BMI }\end{array}$ \\
\hline Clinical data & $\begin{array}{l}\text { Location of latest VTE } \\
\text { Thromboembolic risk factors present } \\
\text { History of VTE or pregnancy losses } \\
\text { History of VTE or early-age stroke/MI } \\
\text { (<50 y) in 1st degree relatives } \\
\text { Known hypercoagulable state in family } \\
\text { Comorbidities present }\end{array}$ \\
\hline Thrombophilia & $\begin{array}{l}\text { Number and result of thrombophilia tests } \\
\text { Antiphospholipid antibodies }\end{array}$ \\
& $\begin{array}{l}\text { Factor V Leiden } \\
\text { Prothrombin G20210A mutation } \\
\text { Antithrombin deficiency } \\
\text { Protein S deficiency } \\
\text { Protein C deficiency } \\
\text { Jak } 2 \text { mutation } \\
\text { Paroxysmal nocturnal hemoglobinuria } \\
\text { Others } \\
\text { Repeat tests and their results } \\
\text { Reasons of incomplete workup }\end{array}$ \\
\hline Management & $\begin{array}{l}\text { Diagnostic workup for VTE } \\
\text { Management plan, and any changes } \\
\text { influenced by thrombophilia results }\end{array}$ \\
\hline data & $\begin{array}{l}\text { Cost of tests } \\
\text { Institutional }\end{array}$ \\
\hline
\end{tabular}

Abbreviations: BMI, body mass index; MI, myocardial infarction; VTE, venous thromboembolism.

aLupus anticoagulant, immunoglobulin ( $\mathrm{g} g$ G and IgM anticardiolipin, IgG and IgM anti- $\beta 2$ glycoprotein I. 
as complete if it included tests for common inherited and acquired thrombophilias that are known to have a reasonably well-defined role in pathogenesis of VTE based on contemporary knowledge. These include factor $\mathrm{V}$ Leiden (FVL), prothrombin G20210A (PT) gene mutation, AT activity level, protein S (PS) activity level, protein C (PC) activity level, and antiphospholipid antibody (APLA) panel. The APLA panel comprised of lupus anticoagulant, immunoglobulin (Ig) G and IgM anticardiolipin antibody, and IgG and IgM anti- $\beta 2$ glycoprotein I antibody.

"Incomplete workup" included (1) Failure to order any of the aforementioned tests. (2) "Wrong timing": testing for PC, PS, and AT activity levels under conditions that might have jeopardized accurate interpretation of results: testing within 1 week of acute thrombosis or during ongoing anticoagulation with warfarin for PC and PS activity levels, heparin for AT activity level, and DOACs for PC, PS, and AT activity levels. 4,13,14 (3) Failure to repeat a positive APLA panel after 12 weeks of initially positive test results. ${ }^{13,14}$ Functional PC, PS, and AT assays were called abnormal if they were out of the normal laboratory ranges. Given that establishing a diagnosis of natural anticoagulant deficiency requires confirmatory repeat testing, physician's assessment, and correlation with the patient's clinical history, the final diagnosis (e.g., PS deficiency) was based on the interpretation of results by the treating hematologist.

Repeated functional/antigenic assays were categorized as: (1) Appropriate/justified, if assays were repeated to confirm initial abnormal results, or to account for "wrong timing" and uninterpretable initial test results. (2) Inappropriate/unjustified, if initial assays were normal, or if assays were repeated during "wrong timing" as specified above. Repetition of any genetic testing was considered unjustified. Criteria for appropriateness of test repeats were based on the recommendations in the review articles by Nakashima and Rogers, Tientadakul et al, and Mahajerin et al. ${ }^{13,15,16}$ Given the uncertainty about the role of monitoring APLA titers in patients with established APS, we did not include the APLA panel in our analysis of appropriateness of repeated testing.

The influence of thrombophilia testing results on patient management was measured as follows: (1) effect on choice on anticoagulant, and (2) effect on duration of anticoagulation. Information on change in patient management was obtained from physician notes that followed thrombophilia testing.

All tests ordered as part of thrombophilia evaluation were included in the calculation of direct cost of the workup. The laboratory charges associated with individual thrombophilia tests were obtained from the Emory University pathology laboratory services and was based on the year 2015 rates (-Table 2). For each thrombophilia test, the tested patients were assigned a cost value that reflected the laboratory charges for the thrombophilia test multiplied by the testordering frequency. For patients who were tested outside the Emory Healthcare System, and for whom we were unable to verify the test-ordering frequency, we made the assumption that they were tested once. One exception was patients who had been given a diagnosis of APS. For such patients, the frequency of ordering the APLA panel was considered as two.

Table 2 Direct medical costs of thrombophilia testing at Emory University Hospitals (in US dollars)

\begin{tabular}{|c|c|c|c|c|}
\hline Test name & $N$ tested patients & Mean cost ${ }^{a}$ & Minimum cost & Maximum cost ${ }^{b}$ \\
\hline Antiphospholipid antibody (APLA) panel & 160 & $\$ 2,846.03$ & $\$ 553.00$ & $\$ 15,398.00$ \\
\hline Factor V Leiden & 159 & $\$ 119.44$ & $\$ 75.00$ & $\$ 648.00$ \\
\hline Prothrombin mutation & 153 & $\$ 86.59$ & $\$ 44.00$ & $\$ 522.00$ \\
\hline Antithrombin level $^{\mathrm{C}}$ & 151 & $\$ 134.98$ & $\$ 54.13$ & $\$ 408.00$ \\
\hline Protein S level $^{\mathrm{C}}$ & 133 & $\$ 182.41$ & $\$ 37.00$ & $\$ 1,062.00$ \\
\hline Protein $\mathrm{C}_{\text {level }}{ }^{\mathrm{C}}$ & 127 & $\$ 160.78$ & $\$ 65.35$ & $\$ 480.00$ \\
\hline Jak 2 mutation & 25 & $\$ 174.41$ & $\$ 80.00$ & $\$ 324.00$ \\
\hline PNH flow cytometry & 22 & $\$ 1,168.45$ & $\$ 138.00$ & $\$ 2,005.00$ \\
\hline Fibrinogen activity level & 77 & $\$ 89.79$ & $\$ 30.00$ & $\$ 104.00$ \\
\hline Homocysteine level & 72 & $\$ 139.41$ & $\$ 60.00$ & $\$ 167.00$ \\
\hline Factor VIII level & 55 & $\$ 207.45$ & $\$ 63.00$ & $\$ 204.00$ \\
\hline Factor IX level & 48 & $\$ 214.28$ & $\$ 67.00$ & $\$ 216.00$ \\
\hline Factor XI level & 47 & $\$ 202.35$ & $\$ 63.00$ & $\$ 204.00$ \\
\hline Lipoprotein a level & 33 & $\$ 32.76$ & $\$ 33.00$ & $\$ 46.00$ \\
\hline MTHFR mutation & 2 & $\$ 119.00$ & $\$ 119.00$ & $\$ 119.00$ \\
\hline
\end{tabular}

Abbreviations: MTHFR, methylenetetrahydrofolate reductase; PNH, paroxysmal nocturnal hemoglobinuria.

Note: Direct medical costs reflect the costs of the testing kit, test-ordering frequency, and any other laboratory costs.

${ }^{a}$ Mean cost $=$ Total cost of testing for each corresponding thrombophilia/Total $N$ of patients tested for this thrombophilia.

${ }^{b}$ Maximum cost reflects the patient who had the highest cost value in each corresponding test. A high cost value could reflect using a more expensive testing kit (e.g., factor V Leiden [FVL] tested in clinic [ $\$ 216.00]$ vs. hospital [\$75.00]), and/or frequent testing that multiplied the total cost for this corresponding thrombophilia.

'The corresponding cost combines the cost of antigen and activity level testing. 
Statistical analysis: We used the chi-square tests for categorical variables and the Kruskal-Wallis test for continuous variables to compare the distribution of variables between tested and nontested patient groups. Costs of thrombophilia workup were summarized using descriptive statistics. All analyses were conducted using SAS, version 9.4 (SAS Institute, Inc, Cary, North Carolina, United States), and SPSS, version 25.0 (IBM SPSS statistics). A $p$-value of $\leq 0.05$ was considered statistically significant.

\section{Results}

Hematology Service database for the year 2015 yielded 522 patients with a final diagnosis of VTE or hypercoagulable state. After excluding duplicates and patients who did not meet the inclusion criteria, 266 eligible patients were identified (-Fig. 1).

Patient characteristics are presented in - Table 3. Of the 266 included patients, 189 (71.1\%) underwent thrombophilia workup. The cohort of patients that underwent thrombophilia testing was more likely to be younger than 40 years (30.9 vs. $18.2 \%$ ), have had prior pregnancy loss (9.0 vs. $0 \%$ ), or known family history of VTE/thrombophilia/early age stroke/myocardial infarction (24.9 vs. 10.4\%). This cohort was less likely to have had provoked VTE (37 vs. $79.2 \%)$, particularly VTE associated with preceding surgery (9.5 vs. $31.2 \%$ ), indwelling venous catheter (7.4 vs. $18.2 \%$ ), or active malignancy (5.8 vs. $41.6 \%$ ). There were no statistically significant differences between the tested and nontested groups in gender, racial distribution, pregnancy, hormonal use, location of clot (visceral vs. nonvisceral), or history of prior VTE. Overall, 53\% of patients with provoked VTE underwent thrombophilia testing.

The pattern of thrombophilia workup, including details of results, is presented in - Fig. $\mathbf{2}$. The most common thrombophilias tested for were APS in 160 (60.1\%), followed closely by FVL in 159 (59.7\%) and PT gene mutation in 153 (57.5\%) patients. Of the 189 tested patients, 84 (44.4\%) fulfilled the criteria for complete thrombophilia workup. Of patients with abnormal functional assays, 9 were tested during the acute thrombosis period and 13 were tested while receiving therapeutic anticoagulation rendering their results uninterpretable. Four patients with positive APLA panel did not undergo repeat testing to determine persistence. Fifty-one (26.9\%) patients tested positive for one or more of the studied thrombophilias.

- Table 4 presents the pattern of repeat testing for the tests included in the "complete thrombophilia" panel. Among the 159 patients tested for FVL and the 153 patients

From the Emory Hematology Service Database, we identified patients diagnosed with and/or treated for VTE in Emory Healthcare from Jan - Dec $2015(N=522)$

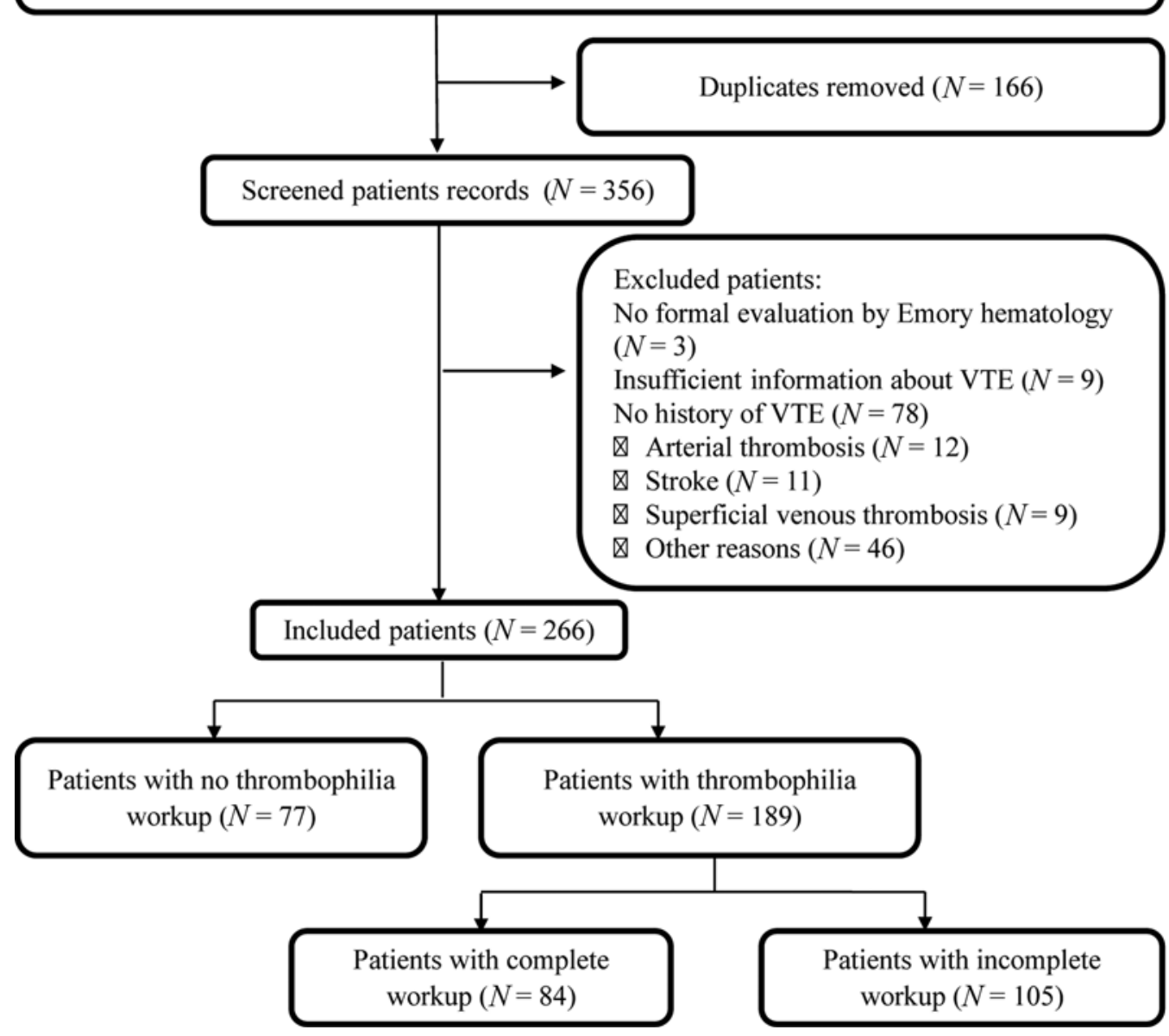

Fig. 1 Flowchart for patient identification. 
Table 3 Patient characteristics

\begin{tabular}{|c|c|c|c|}
\hline Characteristics & $\begin{array}{l}\text { Patients with } \\
\text { thrombophilia } \\
\text { workup }(N=189)\end{array}$ & $\begin{array}{l}\text { Patients without } \\
\text { thrombophilia } \\
\text { workup }(N=77)\end{array}$ & $p$-Value \\
\hline Age at evaluation; median (range) & $52(19-88)$ & $61(20-85)$ & $<0.001$ \\
\hline Age at first VTE; median (range) & $50(14-87)$ & $59(20-85)$ & $<0.001$ \\
\hline Age at first $V T E<40 y$ & $58(30.9)$ & $14(18.2)$ & 0.035 \\
\hline \multicolumn{4}{|l|}{ Sex } \\
\hline Male & $86(45.5)$ & $43(55.8)$ & 0.126 \\
\hline BMI; median (range) & $29.7(14.2-58.7)$ & $28.7(13.5-55.0)$ & 0.213 \\
\hline \multicolumn{4}{|l|}{ Race } \\
\hline White & $92(48.7)$ & $43(55.8)$ & 0.437 \\
\hline African American & $77(40.7)$ & $29(37.7)$ & \\
\hline Other $^{\mathrm{a}}$ & $20(10.6)$ & $5(6.5)$ & \\
\hline Provoked VTE ${ }^{\mathrm{b}}$ & $70(37.0)$ & $61(79.2)$ & $<0.001$ \\
\hline Preceding surgery & $18(9.5)$ & $24(31.2)$ & $<0.001$ \\
\hline Preceding nonsurgical hospitalization/ immobilization ${ }^{c}$ & $23(12.2)$ & $9(11.7)$ & 0.913 \\
\hline Hormone associated (estrogen therapy) & $15(7.9)$ & $6(7.8)$ & 0.384 \\
\hline Pregnancy/Puerperium associated & $6(3.2)$ & $1(1.3)$ & 0.254 \\
\hline Indwelling venous catheter associated $^{d}$ & $14(7.4)$ & $14(18.2)$ & 0.009 \\
\hline Prior VTE & $62(32.8)$ & $21(27.3)$ & 0.377 \\
\hline Prior pregnancy loss(es) & $17(9.0)$ & $0(0.0)$ & 0.003 \\
\hline \multicolumn{4}{|l|}{ High risk comorbidities } \\
\hline Active malignancy & $11(5.8)$ & $32(41.6)$ & $<0.001$ \\
\hline Chronic inflammatory disorders ${ }^{\mathrm{e}}$ & $13(6.9)$ & $2(2.6)$ & 0.170 \\
\hline Nephrotic syndrome & $3(1.6)$ & $0(0.0)$ & 0.266 \\
\hline Congestive heart failure & $8(4.2)$ & $7(9.1)$ & 0.119 \\
\hline Stroke/TIA & $11(5.8)$ & $1(1.3)$ & 0.107 \\
\hline $\begin{array}{l}\text { Family history of VTE, early age stroke/MI or } \\
\text { known thrombophilia }\end{array}$ & $47(24.9)$ & $8(10.4)$ & 0.008 \\
\hline \multicolumn{4}{|l|}{ Most recent VTE location } \\
\hline Visceral DVT & $32(16.9)$ & $7(9.1)$ & $0.101^{f}$ \\
\hline \multicolumn{4}{|l|}{ Nonvisceral } \\
\hline Lower extremity DVT only & $52(27.5)$ & $29(37.7)$ & \\
\hline Upper extremity DVT only & $8(4.2)$ & $13(16.9)$ & \\
\hline Pulmonary embolism only & $55(29.1)$ & $15(19.5)$ & \\
\hline$>1 \mathrm{clot}$ & $51(27.0)$ & 15 (19.5) & \\
\hline
\end{tabular}

Abbreviations: DVT, deep venous thrombosis; MI, myocardial infarction; TIA, transient ischemic attack; VTE, venous thromboembolism.

Note: Categorical variables were presented as $N(\%)$. Bold values reflect significant $p<0.1$.

${ }^{a}$ Other races include Asian, and Hispanic, and no available race.

${ }^{\mathrm{b}}$ Transient factors do not include active malignancy, chronic inflammatory conditions, or chronic infections.

Includes fractures leading to immobilization for at least 3 days.

${ }^{d}$ Catheter associated includes VTE associated with central line, left ventricular assist device, pacemaker, or implantable cardioverter-defibrillator.

${ }^{\text {e} C h r o n i c ~ i n f l a m m a t o r y ~ d i s o r d e r s ~ i n c l u d e ~ i n f l a m m a t o r y ~ b o w e l ~ d i s e a s e, ~ s y s t e m i c ~ l u p u s ~ e r y t h e m a t o s u s, ~ a n d ~ v a s c u l i t i s . ~}$

${ }^{\mathrm{f}} \mathrm{p}$-Value for visceral versus nonvisceral clot locations.

tested for PT G20210 mutation, genetic testing was repeated in $17(10.7 \%)$ and 18 (11.8\%) patients, respectively. Nine patients were tested for a third time. There was no significant correlation between the initial genetic test result and decision to repeat testing. On the other hand, the AT/PS/PC test repeats occurred more frequently in response to an abnormal (deficient/uninterpretable) rather than a normal initial test result. Of the 83 patients found to have abnormal AT/PS/PC assays, 31 (37.3\%) had repeat testing for confirmation. Seven patients were tested three times. From all the testing 


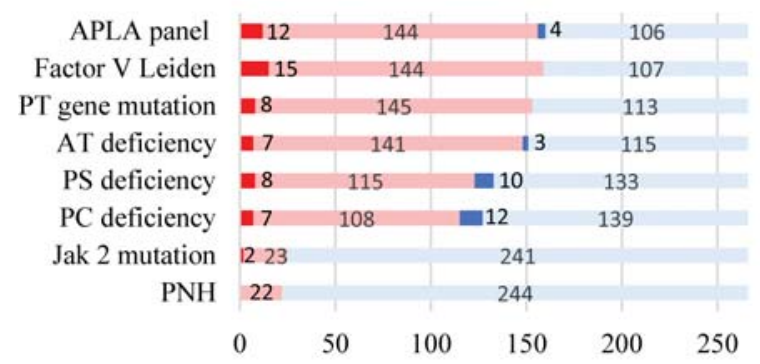

A $\approx$ Positive result $\equiv$ Negative result $₫$ Uninterpretable $\sim$ Not ordered $\mathbf{B}$

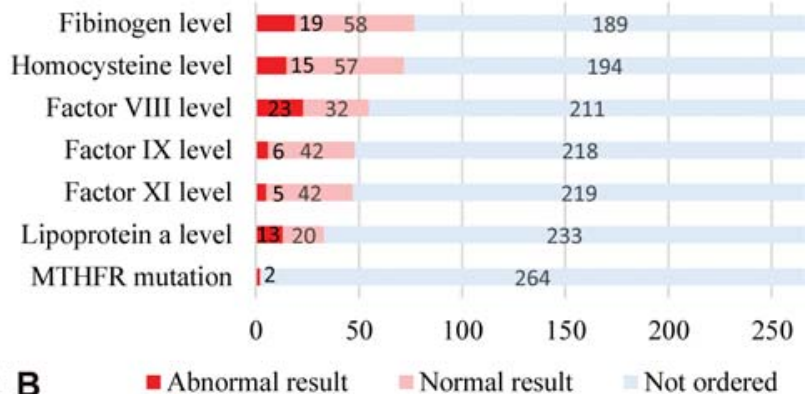

- Abnormal result $\quad$ Normal result $\quad$ Not ordered

Fig. 2 Results of thrombophilia workup in all patients $(N)$. (A) Thrombophilias with reasonably well-defined role in pathogenesis of venous thromboembolism (VTE) based on contemporary knowledge. Uninterpretable APLA testing indicates abnormal APLA panels that were not repeated. Uninterpretable AT, PS, and PC tests include tests that were performed under conditions that jeopardized accurate interpretation of results such as acute thrombosis or ongoing anticoagulation. (B) Other thrombophilias. APLA, antiphospholipid antibody; AT, antithrombin. MTHFR, methylenetetrahydrofolate reductase; PC, protein C; PNH, paroxysmal nocturnal hemoglobinuria; PS, protein S; PT, prothrombin.

performed, hereditary AT/PS/PC deficiency was eventually diagnosed in 22 cases. The appropriateness of thrombophilia test repeats as determined by our predefined criteria is illustrated in - Fig. 3. Twenty-six of the 55 repeated AT/PS/ PC assays were deemed inappropriate because initial assays were normal or assays were repeated during "wrong timing" per criteria described in the "Methods" section.

Thrombophilia testing influenced patient management in 32 (16.9\%) of the 189 tested patients (-Table 5). Results of thrombophilia testing influenced choice of anticoagulant in 6 , duration of anticoagulation in 25 , and both choice and duration of anticoagulation in 1 patient (details provided in footnotes of $\mathbf{- T a b l e ~} \mathbf{5}$ ). Clinical management was modified in 7 of the 12 patients with positive APLA in our study; Warfarin was chosen over DOACs in four patients, and anticoagulation was extended indefinitely in the other three patients. In 19 patients who had negative workup or tested positive only for a low-risk thrombophilia (e.g., heterozygous FVL), physicians felt encouraged to carry out a safe discontinuation trial. The outcome of this trial was guided by the D-dimer levels measured 1 month after discontinuation of anticoagulation therapy. Of the remaining patients (83.1\%) whose management was not affected by thrombophilia testing, 6 were lost to follow-up and 2 refused further management changes. Anticoagulation therapy was extended in 12 patients despite negative thrombophilia workup because of high D-dimer levels, high residual clot burden, persistent symptoms, or persistent risk factors.

In our cohort of 266 patients, we calculated that the direct cost of thrombophilia testing was $\$ 2,364.32$ per patient, $\$ 12,331.55$ to diagnose one positive case, and $\$ 19,653.41$ per patient-management affected. This amounted to a total annual expenditure of $\$ 628,909.12$ toward the direct cost of thrombophilia testing. - Table 6 presents the detailed direct costs of thrombophilia workup in the entire cohort as well as in the patient subgroup that underwent thrombophilia workup.

\section{Discussion}

We have presented the pattern and clinical utilization of thrombophilia testing, and the direct cost associated with such tests at our institution. We noticed significant variability in selection of patients for thrombophilia testing, and in the panel of tests ordered in individual patients. Although the direct cost of testing was high, results impacted management decisions in very few patients.

The question of who should be tested for thrombophilia remains a matter of heightened debate. While there are no clinical trials available to provide evidence-based guidance on the issue, consensus guidelines from national and international academic societies recommend against indiscriminate testing for hereditary/acquired thrombophilia in patients with VTE. ${ }^{2,17,18}$ A significant proportion of patients in our cohort underwent thrombophilia testing, with younger patients, those with unprovoked thrombosis, known family history of thrombosis or thrombophilia, or without a personal history of cancer being more likely to have undergone testing. The patient selection in our cohort seems to follow the "testing selectivity" recommended by some guidelines. For example, the British Society of Haematology recommends testing in patients who present with VTE at an early age ( $<40$ years old), recurrent VTE, or with family history of unprovoked thrombosis in 1 st degree relatives. ${ }^{18}$ Nonetheless, there was a noticeable proportion of patients in our cohort that underwent thrombophilia testing in the absence of any of the aforementioned indications. Fifty-three percent of patients with provoked VTE underwent thrombophilia testing in our study, corresponding to $37 \%$ of total tested patients.

It is well known that the relative risk for VTE associated with different clinical thromboembolic provoking factors is variable. Surgical hospitalization, for example, is associated with a significantly increased risk for VTE (odds ratio $=18.95$, 95\% confidence interval 9.22-38.97), whereas estrogen use increases this risk by only 1.81 times. The latter is, therefore, considered a "weak" clinical risk factor., 19,20 This could explain, in part, some of the testing decisions among patients with provoked VTE in our cohort. Similar pattern was reported in a large cohort of 1,314 patients with VTE by Meyer et al; the presence of "weak" thromboembolic risk factors (e.g., pregnancy or hormone use) did not seem to affect the decision of thrombophilia testing, whereas active 
Table 4 Pattern of repeat thrombophilia testing

\begin{tabular}{|c|c|c|c|c|c|c|c|c|}
\hline \multirow[t]{3}{*}{ First test results } & \multirow[t]{3}{*}{$N$} & \multicolumn{3}{|c|}{ Was test repeated? } & \multicolumn{4}{|c|}{ Number of test repeats } \\
\hline & & Yes & No & & 2nd test & $\begin{array}{l}\text { Positive } \\
\text { result }\end{array}$ & 3rd test & $\begin{array}{l}\text { Positive } \\
\text { result }\end{array}$ \\
\hline & & $N(\%)$ & $N(\%)$ & $p$-Value & $N$ & $N(\%)$ & $N$ & $N(\%)$ \\
\hline \multicolumn{9}{|l|}{ FVL } \\
\hline Positive & 15 & $2(13.3)$ & $13(86.7)$ & 0.728 & 2 & & 0 & \\
\hline Negative & 144 & $15(10.4)$ & $129(89.6)$ & & 12 & & 3 & \\
\hline Total & 159 & $17(10.7)$ & $142(89.3)$ & & 14 & $2 / 14(14.3 \%)$ & 3 & $0 / 3(0.0 \%)$ \\
\hline \multicolumn{9}{|l|}{ PT mutation } \\
\hline Positive & 8 & $1(12.5)$ & $7(87.5)$ & 0.947 & 1 & & 0 & \\
\hline Negative & 145 & $17(11.7)$ & $128(88.3)$ & & 11 & & 6 & \\
\hline Total & 153 & $18(11.8)$ & $135(88.2)$ & & 12 & $1 / 12(8.3 \%)$ & 6 & $0 / 6(0.0 \%)$ \\
\hline \multicolumn{9}{|l|}{ AT level } \\
\hline Deficient $^{\mathrm{a}}$ /Uninterpretable & 20 & $10(50.0)$ & $10(50.0)$ & $<0.001$ & 10 & & 0 & \\
\hline Normal & 131 & $14(10.7)$ & $117(89.3)$ & & 8 & & 6 & \\
\hline Total & 151 & $24(15.9)$ & $127(84.1)$ & & 18 & $2 / 18(11.1 \%)$ & 6 & $0 / 6(0.0 \%)$ \\
\hline \multicolumn{9}{|l|}{ PS level } \\
\hline Deficient ${ }^{\mathrm{b}} /$ Uninterpretable & 25 & $6(24.0)$ & $19(76.0)$ & 0.002 & 3 & & 3 & \\
\hline Normal & 108 & $5(4.6)$ & $103(95.4)$ & & 4 & & 1 & \\
\hline Total & 133 & $11(8.3)$ & $122(91.7)$ & & 7 & $1 / 7(14.3 \%)$ & 4 & $1 / 4(25.0 \%)$ \\
\hline \multicolumn{9}{|l|}{ PC level } \\
\hline Deficient ${ }^{\mathrm{C}} /$ Uninterpretable & 38 & $15(39.5)$ & $23(60.5)$ & $<0.001$ & 11 & & 4 & \\
\hline Normal & 89 & $5(5.6)$ & $84(94.4)$ & & 4 & & 1 & \\
\hline Total & 127 & $20(15.7)$ & $107(84.3)$ & & 15 & $1 / 15(6.7 \%)$ & 5 & $2 / 5(40.0 \%)$ \\
\hline \multicolumn{9}{|l|}{ Jak 2 mutation } \\
\hline Positive & 2 & 0 & $2(100.0)$ & $\mathrm{N} / \mathrm{A}$ & 0 & & 0 & \\
\hline Negative & 23 & 0 & $23(100.0)$ & & 0 & & 0 & \\
\hline Total & 25 & 0 & $25(100.0)$ & & 0 & $\mathrm{~N} / \mathrm{A}$ & 0 & $\mathrm{~N} / \mathrm{A}$ \\
\hline \multicolumn{9}{|l|}{ PNH flow cytometry } \\
\hline Positive & 0 & 0 & $0(100.0)$ & $\mathrm{N} / \mathrm{A}$ & 0 & & 0 & \\
\hline Negative & 22 & 0 & $22(100.0)$ & & 0 & & 0 & \\
\hline Total & 22 & 0 & $22(100.0)$ & & 0 & $\mathrm{~N} / \mathrm{A}$ & 0 & $\mathrm{~N} / \mathrm{A}$ \\
\hline
\end{tabular}

Abbreviations: AT, antithrombin; FVL, factor V Leiden; N/A, not applicable; PC, protein C; PNH, paroxysmal nocturnal hemoglobinuria; PS, protein S; PT, prothrombin.

Notes: AT/PS/PC antigen and activity levels were called "deficient" if they were below the low normal value in the reference laboratory range. Bold values reflect significant $p<0.1$.

${ }^{a}$ The normal range for AT antigen level was 214-318, and the normal range for AT activity level was $83-128 \%$.

bThe normal range for PS antigen level was 70-155, and the normal range for PS activity level was $64-149 \%$.

'The normal range for PC antigen level was 70-140, and the normal range for PC activity level was 90-183\%.

cancer, recent hospitalization, lung disease, or indwelling venous catheters were significantly associated with no thrombophilia testing. ${ }^{21}$

Unnecessary or unreliable thrombophilia testing has been reported by many researchers, and reflects the persistent problem of poor patient and timing selection. ${ }^{21-23}$ In our cohort, $11.6 \%$ of all ordered tests were uninterpretable because of being confounded by acute clot or concurrent anticoagulation. Other researchers have observed even higher proportions of unreliable testing. In one study, $35.2 \%$ of patients were tested within 7 days of index VTE, and in another, 63\% of abnormal PS and PC test results were attributed to concurrent anticoagulation therapy. ${ }^{15,21,22}$ These observations raise concerns regarding the high likelihood of inaccurate results and their impact on the cost and management decisions for patients with VTE in our daily practice.

Thrombophilia testing is expensive, with many studies indicating a cost burden rather than a benefit of testing, because of lack of universal guidelines and the magnitude of inappropriate testing. ${ }^{24-27}$ In our cohort of 266 patients, we 


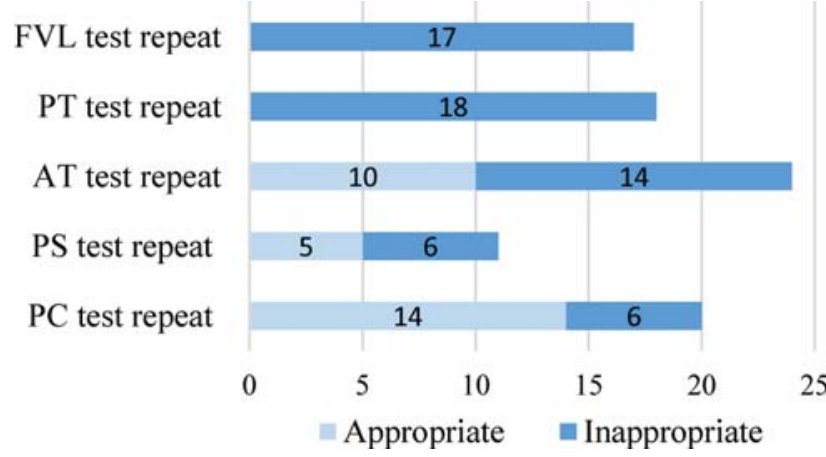

Fig. 3 Appropriateness of thrombophilia test repeats $(N)$. AT, antithrombin; FVL, factor V Leiden; PC, protein C; PS, protein S; PT, prothrombin.
Table 6 Cost of thrombophilia workup (US dollar)

\begin{tabular}{|l|l|l|}
\hline & $\begin{array}{l}\text { Cost per } \\
\text { patient }\end{array}$ & $\begin{array}{l}\text { Cost per positive } \\
\text { patient }\end{array}$ \\
\hline All patients $(N=266)$ & $2,364.32$ & $12,331.55$ \\
\hline $\begin{array}{l}\text { Patients with } \\
\text { workup }(N=189)\end{array}$ & $3,308.43$ & $12,260.65$ \\
\hline $\begin{array}{l}\text { Patients with incomplete } \\
\text { workup }(N=105)\end{array}$ & $2,701.69$ & $12,894.43$ \\
\hline $\begin{array}{l}\text { Patients with complete } \\
\text { workup }(N=84)\end{array}$ & $4,066.84$ & $11,779.81$ \\
\hline
\end{tabular}

Note: Cost per patient $=$ total cost/total $N$ of patients. Cost per positive patient $=$ total cost $/$ total $N$ of positive patients. calculated that the direct cost of thrombophilia testing was $\$ 2,364.32$ per patient and $\$ 19,653.41$ per one management change. These values do not account for the cost and complications downstream of extended anticoagulation in patients with potentially false positive results (i.e., lack of confirmatory repeat test) or for the anxiety of being diagnosed with a hereditary disorder. ${ }^{28}$

Ideally, the decision to perform thrombophilia testing in patients with VTE should be based, in part, on whether results will influence clinical management. In our cohort, thrombophilia testing influenced the duration and/or choice of anticoagulation in only $16.9 \%$ of all tested patients. For VTE provoked by major temporary risk factors (e.g., surgical hospitalization), guidelines recommend a maximum of 3 months of therapeutic anticoagulation. ${ }^{5}$ In patients with unprovoked VTE in whom extended anticoagulation is considered, the value of thrombophilia testing is rather controversial. It is important here to emphasize that a negative thrombophilia workup does not reduce the risk of VTE recurrence in patients with unprovoked VTE. ${ }^{29}$ Therefore, deciding the duration of anticoagulation in these cases rests on the assessment of other pertinent factors such as patient characteristics, symptoms, D-dimer level, residual clot burden, and risk of bleeding. ${ }^{30}$ One reasonable and frequently observed utility of thrombophilia testing in our patients with unprovoked VTE was to use the results to decide whether a discontinuation trial could be safe in patients who desired limited duration of anticoagulation or whose risk of bleeding was moderate.

As far as choice of anticoagulant is concerned, the only thrombophilia that has a bearing is high-risk APS (APS with triple antibody positivity and/or arterial thrombosis). ${ }^{3,9}$ Recent studies have indicated that DOACs may be inferior to warfarin in high-risk APS patients due to a higher risk of arterial events with DOACs. ${ }^{31}$ In our cohort, choice of anticoagulant was based on APLA results in seven patients; warfarin was chosen over DOACs in four because of positive results, and three patients were switched from warfarin to a DOAC after their APLA panels resulted negative. Therefore, there may be clinical utility for early testing for APS in patients with unprovoked VTE or VTE associated with "weak" clinical risk factors while making a decision about appropriate type of anticoagulant for individual patients.

Our study is limited mainly by its retrospective nature and reliance on chart documentation of relevant positive and negative histories. It is our standard hematology practice at Emory University Hospitals to include all pertinent positive medical history, including information on pregnancy losses, in the initial inpatient consult/outpatient visit note for patients who were referred for thrombosis. Patient information was collected from the initial visit note as well as notes from subsequent visits with Emory Hematology. Patients with insufficient information on the index VTE were excluded from the study ( $N=9$, - Fig. 1$)$. This allowed completion of

Table 5 Effect of thrombophilia workup on management

\begin{tabular}{|l|l|l|l|l|}
\hline \multicolumn{2}{|l|}{$\begin{array}{l}\text { Was management plan } \\
\text { changed? } N(\%)\end{array}$} & \multicolumn{2}{|l|}{ How was management plan changed? } \\
\cline { 3 - 5 } & Choice of AC, $\boldsymbol{N}(\%)$ & Duration of $\mathrm{AC}, \boldsymbol{N}(\%)$ & Choice and duration of $\mathrm{AC}, \boldsymbol{N}(\%)$ \\
\hline Yes & $32 / 189^{\mathrm{a}}(16.9)$ & $6 / 32^{\mathrm{b}}(18.8)$ & $25 / 32^{\mathrm{C}}(78.1)$ & $1 / 32^{\mathrm{d}}(3.1)$ \\
\hline No & $157 / 189(83.1)$ & N/A & N/A & N/A \\
\hline
\end{tabular}

Abbreviations: AC, anticoagulation; APS, antiphospholipid syndrome; AT, antithrombin; FVL, factor V Leiden; N/A, not applicable; PS, protein S. ${ }^{\mathrm{a}} 189$ represent total number of patients who underwent any thrombophilia workup.

${ }^{b}$ Three patients with negative antiphospholipid antibody (APLA) panel were switched from warfarin to direct oral anticoagulants. In another three patients, warfarin was continued/started because of positive APS workup. One patient with heterozygous FVL and 18 patients with negative workup were taken off anticoagulation, after a safe discontinuation trial.

${ }^{\mathrm{C}}$ Anticoagulation was extended in three patients with a positive APS antibody panel, one patient with PS deficiency, one patient with elevated factor VIII and IX, and one patient with AT deficiency.

In one patient with a triple positive APLA panel, anticoagulation was switched to warfarin and continued indefinitely. 
data to the best of our knowledge. For patients who received part of their thrombosis care outside Emory Healthcare, we were not able to verify the timing or the number of times a particular test was ordered for these patients. We reviewed EMR for patients who presented to the Emory Hematology service in 2015, and therefore, utilized the average charges for individual thrombophilia tests in the same year. In some cases, however, testing was done in preceding or following years, or outside of Emory Healthcare System, which may have undermined the precision of our calculated costs. Nevertheless, our results provide an estimate of total annual expenditure pertaining to thrombophilia testing and were close to that reported in other contemporary analyses. ${ }^{28}$ While we realize that the cost of medical care including indirect costs and downstream effects of medical decisions have an impact on the overall cost-effectiveness of thrombophilia testing, this was beyond the scope of our inquiry.

\section{Conclusion}

Our results highlight the variability in selection of patients and panel of tests for thrombophilia testing among patients with VTE. The direct cost associated with thrombophilia testing was high, though the results were utilized in clinical decision making in very few patients. With guidelines advocating selective use of thrombophilia testing and attention to potential impact of test results in patient management, we propose the need for measures at institutional levels to improve thrombophilia test-ordering practices. These strategies could include development of local guidelines, continuing medical education, and the implementation of clinical decision support systems within the electronic medical charts.

\section{Authors' Contributions}

M.G.: conceptualization, study design, data extraction and curation, manuscript drafting, and editing; E.C.: study and experimental design, data extraction, curation and analysis, and manuscript review; M.A.T.E.: experimental design, data curation and analysis, data presentation, manuscript drafting, and editing; I.B.: conceptualization, study design, and manuscript review.

\section{Funding}

This work was supported by the Hungarian National Research Development and Innovation Office (NFKI) grant OTKA-K19_131945 (I.B.).

\section{Conflict of Interest}

M.G. received advisory board honoraria from Hema biologics, Pharmacyclics LLC, Agios, and Pfizer. E.C., M.A.T.E., and I.B. have no conflict of interest to disclose.

\section{References}

1 Franchini M, Veneri D, Salvagno GL, Manzato F, Lippi G. Inherited thrombophilia. Crit Rev Clin Lab Sci 2006;43(03): 249-290
2 Stevens SM, Woller SC, Bauer KA, et al. Guidance for the evaluation and treatment of hereditary and acquired thrombophilia. J Thromb Thrombolysis 2016;41(01):154-164

3 Bertoletti L, Benhamou Y, Béjot Y, et al. Direct oral anticoagulant use in patients with thrombophilia, antiphospholipid syndrome or venous thrombosis of unusual sites: a narrative review. Blood Rev 2018;32(04):272-279

4 Pruthi RK. Optimal utilization of thrombophilia testing. Int J Lab Hematol 2017;39(Suppl 1):104-110

5 Kearon C, Akl EA, Ornelas J, et al. Antithrombotic therapy for VTE disease: CHEST Guideline and Expert Panel Report. Chest 2016; 149(02):315-352

6 National Institute for Health and Clinical Excellence. Venous Thromboembolic Diseases: Diagnosis, Management and Thrombophilia Testing Clinical Guideline [CG144]. London, UK: National Institute for Health and Clinical Excellence; 2012

7 Christiansen SC, Cannegieter SC, Koster T, Vandenbroucke JP, Rosendaal FR. Thrombophilia, clinical factors, and recurrent venous thrombotic events. JAMA 2005;293(19):2352-2361

8 Mannucci PM, Franchini M. Classic thrombophilic gene variants. Thromb Haemost 2015;114(05):885-889

9 Elsebaie MAT, van Es N, Langston A, Büller HR, Gaddh M. Direct oral anticoagulants in patients with venous thromboembolism and thrombophilia: a systematic review and meta-analysis. J Thromb Haemost 2019;17(04):645-656

10 Elsebaie MAT, Van Es N, Langston A, Büller HR, Gaddh M. Followup to comment on "Direct Oral Anticoagulants in Patients with Venous Thromboembolism and Thrombophilia: Systematic Review and Meta-Analysis". J Thromb Haemost 2019;17(06): 1007-1009

11 Simpson EL, Stevenson MD, Rawdin A, Papaioannou D. Thrombophilia testing in people with venous thromboembolism: systematic review and cost-effectiveness analysis. Health Technol Assess 2009;13(02):iii, ix-x, 1-91

12 Kearon C, Ageno W, Cannegieter SC, Cosmi B, Geersing G-J, Kyrle PA; Subcommittees on Control of Anticoagulation, and Predictive and Diagnostic Variables in Thrombotic Disease. Categorization of patients as having provoked or unprovoked venous thromboembolism: guidance from the SSC of ISTH. J Thromb Haemost 2016; 14(07):1480-1483

13 Nakashima MO, Rogers HJ. Hypercoagulable states: an algorithmic approach to laboratory testing and update on monitoring of direct oral anticoagulants. Blood Res 2014;49(02):85-94

14 Petrilli CM, Heidemann L, Mack M, Durance P, Chopra V. Inpatient inherited thrombophilia testing. J Hosp Med 2016;11(11): 801-804

15 Tientadakul P, Chinthammitr Y, Sanpakit K, Wongwanit C, Nilanont Y. Inappropriate use of protein $C$, protein $S$, and antithrombin testing for hereditary thrombophilia screening: an experience from a large university hospital. Int J Lab Hematol 2011;33(06): 593-600

16 Mahajerin A, Obasaju P, Eckert G, Vik TA, Mehta R, Heiny M. Thrombophilia testing in children: a 7 year experience. Pediatr Blood Cancer 2014;61(03):523-527

17 Pernod G, Biron-Andreani C, Morange P-E, et al; French group on haemostasis and thrombosis; French Society of vascular medicine. Recommendations on testing for thrombophilia in venous thromboembolic disease: a French consensus guideline. J Mal Vasc 2009;34(03):156-203

18 Baglin T, Gray E, Greaves M, et al; British Committee for Standards in Haematology. Clinical guidelines for testing for heritable thrombophilia. Br J Haematol 2010;149(02):209-220

19 Heit JA, Silverstein MD, Mohr DN, Petterson TM, O'Fallon WM, Melton LJ III. Risk factors for deep vein thrombosis and pulmonary embolism: a population-based case-control study. Arch Intern Med 2000;160(06):809-815

20 Anderson FA Jr, Spencer FA. Risk factors for venous thromboembolism. Circulation 2003;107(23, Suppl 1):I9-I16 
e162 Thrombophilia Testing in Venous Thromboembolism Gaddh et al.

21 Meyer MR, Witt DM, Delate T, et al. Thrombophilia testing patterns amongst patients with acute venous thromboembolism. Thromb Res 2015;136(06):1160-1164

22 Somma J, Sussman II, Rand JH. An evaluation of thrombophilia screening in an urban tertiary care medical center: a "real world" experience. Am J Clin Pathol 2006;126(01):120-127

23 Favaloro EJ, Mohammed S, Pati N, Ho MY, McDonald D. A clinical audit of congenital thrombophilia investigation in tertiary practice. Pathology 2011;43(03):266-272

24 O'Brien SH, Smith KJ. Using thrombophilia testing to determine anticoagulation duration in pediatric thrombosis is not costeffective. J Pediatr 2009;155(01):100-104

25 Mou E, Kwang H, Hom J, et al. Magnitude of potentially inappropriate thrombophilia testing in the inpatient hospital setting. J Hosp Med 2017;12(09):735-738

26 Wu O, Robertson L, Twaddle S, et al; Thrombosis: Risk and Economic Assessment of Thrombophilia Screening (TREATS) Study. Screening for thrombophilia in high-risk situations: a meta-analysis and costeffectiveness analysis. Br J Haematol 2005;131(01):80-90

$27 \mathrm{Wu} \mathrm{O}$, Greer IA. Is screening for thrombophilia cost-effective? Curr Opin Hematol 2007;14(05):500-503

28 Shen Y-M, Tsai J, Taiwo E, et al. Analysis of thrombophilia test ordering practices at an academic center: a proposal for appropriate testing to reduce harm and cost. PLoS One 2016;11(05):e0155326

29 Masuda EM, Lee RW, Okazaki IJ, Benyamini P, Kistner RL. Thrombophilia testing has limited usefulness in clinical decision-making and should be used selectively. J Vasc Surg Venous Lymphat Disord 2015;3(02):228-235

30 Couturaud F. Guided duration of anticoagulation after unprovoked venous thromboembolism using D-dimer testing. Eur Respir J 2016;47(05):1313-1314

31 Pengo V, Denas G, Zoppellaro G, et al. Rivaroxaban vs warfarin in high-risk patients with antiphospholipid syndrome. Blood 2018; 132(13):1365-1371 